\title{
OPTIMIZATION OF STERILE MALE RATIO OF ORIENTAL FRUIT FLY, Bactrocera dorsalis (HENDEL)
}

\author{
A. Zahan ${ }^{* 1}$, M. A. Latif ${ }^{2}$, M. A. Hossain ${ }^{3}$, S. A. Khan ${ }^{3}$ and M. J. Rahman ${ }^{2}$ \\ Department of Entomology, Sher-e-Bangla Agricultural University \\ Sher-e-Bangla Nagar, Dhaka-1207, Bangladesh
}

\begin{abstract}
The study was conducted to establish the Sterile Insect Technique (SIT) method of controlling oriental fruit fly, Bactrocera dorsalis (Hendel). The sterile male ratio of oriental fruit fly was optimized for possible field application of Sterile Insect Technique. Several batches of 5 and 6-dayold pupae were irradiated at 30, 40,50 and 60 Gray (Gy) to optimize the radiation dose of sterilization. Highest pupal duration and abnormal adults were found after imposing gamma radiation in 5-day-old pupae treated with 60 Gy dose. Radiation significantly increased the nonemergence percentage and decreased the normal emergence of oriental fruit fly. The sterilizing doses were recorded as 60 Gy for 5-dayold pupae. Fixed number of virgin females of oriental fruit flies were allowed to mate with unirradiated and irradiated males at 1:1, 1:2, 1:5 and 1:9 ratios in different cages to find out the rate of suppression against wild population. Minimum pupal recovery and percent normal emergence observed at 1:9 ratio. Deformed and undeveloped (not emerged) population increased with the ratio of irradiated males. The 1:9 ratio was found as the best for suppression of wild population of $B$. dorsalis.
\end{abstract}

Keywords: Irradiation, ratio optimization, sterile insect technique (SIT), sterile insect release method (SIRM)

\section{INTRODUCTION}

Fruit flies are responsible for taking a heavy toll of various types of fruits and vegetables. The oriental fruit fly is a very important group of pests for many countries due to their potential to cause damage in fruits, vegetables and to their potential to restrict access to international markets for plant products that can host

\footnotetext{
*Corresponding author email: asrinzahan@yahoo.com

${ }^{1}$ Senior Sector Specialist, Bangladesh Rural Advancement Committee, Dhaka, Bangladesh

${ }^{2}$ Department of Agronomy, Sher-e-Bangla Agricultural University, Sher-e-Bangla Nagar, Dhaka-1207, Bangladesh

${ }^{3}$ Insect Biotechnology Division (IBD), Institute of Food and Rediation Biology (IFRB), Atomic Energy Research

Establishment (AERE), Savar, Dhaka, Bangladesh
}

Received: 26.05 .2015 
fruit flies. Flies in the genus Bactrocera are of particular concern in most part of Asia and Australia, where they constitute a significant threat to agricultural resources (Kinnear et al., 1998 and Kim et al., 1999). The genus Bactrocera of the family Tephritidae having more or less 440 species is distributed principally in tropical Asia, the South Pacific and Australia (White and Elson-Harris, 1994). Eighty seven species of the genus Bactrocera in India caused heavy damage to fruits and vegetables in Asia (Nagappan et al., 1971). In Bangladesh oriental fruit fly, Bactrocera dorsalis is recognized as a destructive pest species where the production of vegetables and fruits is much less than the requirement (Add ref.). A preliminary survey showed that a large amount of quality fruits including apricot, banana, mango, guava, papaya, avocado, citrus, litchi, pineapple, and seasonal vegetables are being lost due to fruit fly infestation in Bangladesh (Alim et al., 2012). A conservative estimate indicated that 10 to $15 \%$ of crops are destroyed by fruit flies every year (Awal, 2005).

The classical use of pesticides has been well-established as a fully dependable weapon in crop protection as it causes environmental pollution and health hazards to the pesticide applicators as well as consumers of agricultural produces. The sterile insect technique (SIT) has been well-established as a successful method of controlling key pests of agricultural importance (Snow, 1988 and Vagras, 1989). SIT is a species specific environmentally safer process and when applied successfully could eradicate the target pests from a definite area (Dowell and Siddiqui, 2000). The sterile insect technique is a method of biological control whereby overwhelming number of sterile insects are released. The released insects are normally male. The sterile male competes with wild male for pairing with female insects, if female mates with a sterile male then it will not produce offspring and thus the next generation population will be reduced. The dosage of radiation applied must have no significant adverse effect on the male's longevity, searching behavior and mating ability (Barry and Morse, 2004). Sterile insects are not self-replicating and therefore cannot be established in the environment. SIT does not introduce exotic species into an ecosystem. Considering the above facts in mind, the experiment was undertaken to optimize the sterile male ratio of oriental fruit fly for suppression of $B$. dorsalis population.

\section{MATERIALS AND METHODS}

The present study was conducted in the laboratory and experimental field of Insect Biotechnology Division (IBD), Institute of Food and Radiation Biology (IFRB), Atomic Energy Research Establishment (AERE), Savar, Dhaka during January 2012 to February 2013.

\section{Stock culture}

About 5,000 adult flies were maintained in steel framed cages $(76 \times 66 \times 76$ $\mathrm{cm})$ covered with wired net. The flies were supplied with protein based artificial diets viz., (i) baking yeast: sugar: water at 1:3:4 ratio, and (ii) casein: yeast extract: sugar at 
1:1:2 ratio. Water was supplied in a conical flask socked with cotton ball. Temperature $\left({ }^{\circ} \mathrm{C}\right)$ and relative humidity $(\mathrm{RH})$ of the rearing room was maintained at $27 \pm 2^{\circ} \mathrm{C}$ and $75 \pm 5 \%$, respectively by using air conditioner (Model No. Movincool Classic Plus 26, USA).

\section{Pupae collection and irradiation}

Eggs were collected and washed with distilled water and sieved with a very fine screen and then measured volumetrically. Larvae of oriental fruit fly were reared in the laboratory using artificial standard larval diet and kept in larger bowls contained 1.5 to $4 \mathrm{~cm}$ thick sawdust used for pupation. Sawdust was sieved and collected pupae were transferred into Petri dishes and irradiated by exposing them to gamma radiation from a radioactive Cobalt-60 source. To optimize the radiation dose of sterilization several batches of 5 and 6-day-old pupae were irradiated at 30, 40, 50 and 60 Gy dose.

\section{Sterility dose determination}

To determine the sterilizing dose 50 male flies were collected from each of 30 , 40, 50 and 60 Gy treated 5-day-old pupae and 50 females were also isolated from control batch and allowed to mate with individual dose treated males in small rearing cages. After 4 days of mating, small pieces of banana were placed in each cage as an oviposition medium. The piece of banana was removed after 24 hours from the adult cage and placed in a small plastic bowl with sawdust for further larval development. After subsequent days the total number of pupa and pupal duration were counted and recorded. This experiment was repeated 5 times. Before adult emergence, 100 pupae were placed in a Petri dish and allowed to emerge freely in a small screened cage. After completing emergence fully emerged, not emerged and deformed flies were counted and the percentage of emerging flies was calculated for each replication. Ten replicates of 100 pupae from each group (originating from different emergence dates) were performed.

\section{Male ratio optimization}

The male adults of $B$. dorsalis emerged from 5-day-old pupae irradiated with 60 Gy gamma radiation was segregated into separate cage. The males were allowed to mate 1:2, 1:5 and 1:9 (normal male: irradiated male) ratios with virgin females in separate adult cages. Control batches were maintained for the experiment. In field cage trials, 9-day-old 300 virgin females with corresponding number of irradiated and unirradiated males were released in the netted area $(25 \times 10 \times 12.5 \mathrm{~cm})$ in the natural environment. A piece of banana $(200 \mathrm{~g})$ was placed in cage as oviposition medium on the $4^{\text {th }}$ day after mating. Ratio between irradiated males and unirradiated males with unirradiated females was optimized by recording the number of pupal recovery, normal adult emergence, deformed flies emergence and nonemergence percentage and compared with each other. 


\section{Statistical analysis}

The recorded data were compiled and tabulated for statistical analysis. Data was analyzed according to Gomez and Gomez (1984) and mean values were compared by Least Significant Difference (LSD) test.

\section{RESULTS AND DISCUSSION}

\section{Sterility Dose Determination of Male Bactrocera dorsalis}

Pupal durations after imposing gamma radiation at different pupal ages are presented in table 1 . In case of 5-day-old pupae, the highest pupal duration was $11.333 \pm 1.52$ days when irradiated with 60 Gy and the lowest was $8 \pm 1$ days in control batch. In case of 6-day-old pupae, the highest pupal duration was $11 \pm 1$ days when irradiated with $60 \mathrm{~Gy}$ and the lowest was $7.667 \pm 0.58$ days in control batch. Between 5-day-old and 6-day-old pupae the highest pupal duration was $11.333 \pm 1.52$ days when irradiated with 60Gy at 5-day-old pupae.

Normal adult emergence, deformed flies emergence and nonemegence flies percentage after imposing gamma radiation at 5-day-old pupae were presented in table 2. The percentage of normal emergence showed that the highest normal emergence $(93.12 \%)$ was obtained from the control batch and the lowest $(0 \%)$ from the pupae irradiated with 60Gy was which significantly differed from those of other radiation doses. After imposing gamma radiation abnormalities consisted mainly of wrinkled wings or wingless that failed to expand upon emergence. The percentage of deformed flies emergence showed that the highest deformed flies emerged $(18.38 \%)$ from the pupae irradiated with $40 \mathrm{~Gy}$ which significantly differed from those of other radiation doses and the lowest deformed flies emerged (1.593\%) from the control batch. The highest percent not emerged flies $(94.44 \%)$ was found from the pupae irradiated with $60 \mathrm{~Gy}$ which was significantly different from those of other radiation doses and the lowest deformed flies emerged (5.287\%) was found from the control batch.

Pupal recoveries recorded at different doses of gamma irradiation are presented in figure 1 . The highest number of pupal recovery $(984 \pm 9.08)$ was found from 6-dayold pupae at control. This happned because not a single of oriental fruit fly emerged from the 5-day-old pupae treated with $60 \mathrm{~Gy}$ which was selected as sterilizing dose. Islam et al. (2012) reported that in case of 5-day-old pupae of fruit fly the sterility percentage was 100 at $60 \mathrm{~Gy}$. The reasons to have reduced number of pupal recovery at higher doses are related to the damage of genetic materials (Heather and Hallman, 2008). Significant prolongation of pupal duration following radiation may be due to hyper stimulation of the mechanism of repair to slightly damaged tissues (Balock et al., 1963). The sterilizing dose for male B. cucurbitae was reported by Balock et al. (1963) and Wadud et al. (2005) as 50-60 Gy which is almost similar to our present findings with $B$. dorsalis. 


\section{Optimization of the ratios between irradiated and unirradiated males for effective field application of SIT}

In field cage trials number of pupae produced from different mating ratios of un-irradiated and irradiated males with the virgin females of Bactrocera dorsalis are shown in table 3 . The results showed that the pupal recovery varied from 1:2, 1:5, 1:9 ratios and control batch. The highest pupal recovery was found at control batch and the lowest pupal recovery was found in 1:9 ratio. In case of normal emergence percentage the highest result was found in control and the lowest in 1:9 ratio (Table 4). The result showed that the number of pupal recovery and normal emergence percentage is inversely correlated with the ratio having high irradiated male oriental fruit fly i.e. the higher the ratio with irradiated males applied, the lower the number of pupal recovery normal emergence percentage observed. The result agrees with Singh et al. (2000) but they worked on melon fruit fly.

Deformed flies percentage and nonemegence percentage of oriental fruit fly from different mating ratios of un-irradiated and irradiated males with virgin females of Bactrocera dorsalis in field cage trials are shown in table 5 and 6 . The result showed that the percent deformed flies and nonemegence varied from 1:2, 1:5, 1:9 ratios and control batch. The highest result was observed at 1:9 ratio and the lowest was found in control. The percent deformed flies and nonemegence at different ratios differed significantly. The result showed that the deformed flies and nonemegence percentage are positively correlated with the ratio having high irradiated male fruit fly i.e. the higher the ratio with irradiated males applied, the higher the percentage of deformed and not emerged flies observed. This finding has a good agreement with the result of Islam et al. (2012) where percentage of normal adult emergence, deformed flies and percent nonemegence of $B$. tau varied significantly at 1:1, 1:2, 1:5 and 1:9 ratios from control batches. Krishnakumar and Maheswari (2007) studied the optimization of wild and sterile male ratio for the suppression of red palm weevil, Rhynchophorus ferrugineus in cotton and reported that the optimal wild to sterile male ratio was 1:10. Islam et al. (2012) optimized normal versus sterile male ratio of $B$. tau and reported that on an average, 534, 235, 118 and 84 pupae were recovered from 1:1, 1:2, 1:5 and 1:9 ratios respectively and the pupal recovery from control group was 3,562. The results agree with that of our present findings though the species was different. According to above parameters, 1:9 ratio (unirradiated male:irradiated male) of oriental fruit fly is apparently an effective ratio for releasing sterilized male fly in nature for possible field application of SIT.

\section{CONCLUSION}

Sterile Insect Technique (SIT) of pest control using area-wide releases of sterile insects was found to be effective in controlling reproduction of oriental fruit fly in a field population. So, it can be concluded that releasing irradiated males of Bactrocera dorsalis treated with $60 \mathrm{~Gy}$ in nature at 1:9 ratio (unirradiated 
male:irradiated male) could be the optimum ratio to suppress the wild population of oriental fruit flies without rendering any adverse impact on agro-ecosystem.

\section{REFERENCES}

Alim, M. A., Hossain, M. A., Khan, A., Khan, S. A., Islam, M. S. and Khalequzzaman, M. 2012. Seasonal variations of melon fly, Bactrocera cucurbitae (Coquillett) (Diptera: Tephritidae) in different agricultural habitats of Bangladesh. ARPN Journal of Agricultural and Biological Science, 7(11): 905-911

Awal, M. A. 2005. Role of Quarantine in the Impot and Export of the Plants and Plants products, Proc. First Natl. Seminer on Use of Irradiation for Quarantine treatment of Fresh fruits and Vegetables. 19 September, 2005. Pp. 5-13. Atomic Energy Centre, Dhaka

Balock, J. W., Burditt, A. K. and Christenson, L. D. 1963. Effects of gamma radiation on various stages of three fruit fly species. Journal of Economic Entomology, 59: 42-46

Barry, J. D., Blessinger, T. and Morse, J. G. 2004. Recapture of sterile Mediterranean fruit flies (Diptera: Tephritidae) in California's preventative release program. Journal of Economic Entomology, 97(5): 1554- 1562

Dowell, R. V., Siddiqui, I. A., Meyer, F. and Spaugy, E. L. 2000. Mediterranean fruit fly preventative release programme in southern California, pp. 369-375. In: Tan, K. H. (ed.). Proceedings: Area-Wide Control of Fruit Flies and Other Insect Pests. International Conference on Area-Wide Control of Insect Pests, and the $5^{\text {th }}$ International Symposium on Fruit Flies of Economic Importance, 28 May-5 June 1998, Penang, Malaysia. Penerbit Universiti Sains Malaysia, Pulau Pinang, Malaysia

Gomez, K. A. and Gomez, A. 1984. Statistical procedure for agricultural research, pp. 1-68

Heather, N. H. and Hallman, G. J. 2008. Pest management and phytosanitary trade barriers, CAB International, Oxfordshire OX108DE, UK, p. 257

Islam, M. Z., Hossain, M. A., Alim, M. A., Khan, M. and Khan, S. A. 2012. Determination of male sterility dose and optimization of male ratios of Pumpkin fly, Bactrocera tau (Walker) (Diptera:Tephritidae) in support of sterile insect technique application. Bangladesh Journal of Entomology, 22(2): 93-101

Kim, T. H., Kim, J. S. and Mun, J. H. 1999. Distribution and bionomics of Bactrocera (Paradacus) depressa (Shriaki) in Chonbuk province. Korean journal of soil zoology, 4: $26-32$

Kinnear, M. W., Bariana, H. S., Sved, J. A. and Frommer, M. 1998. Polymorphic microsatellite markers for population analysis of a tephritid pest species, Bactrocera tryoni. Molecular Ecology, 7: 1489-1495

Krishnakumar, R. and Maheswari, P. 2007. Area-wide control of insect pests. Springer Publisher, The Netherlands, p. 485

Nagappan, K., Kamalnathan, S., Santharaman, T. and Ayyasamy, M. K. 1971. Insecticidal trials for the control of the melon fruit fly, Dacus cucurbitae Coq. infesting snake gourd, Trichosanthes anguina. The Madras Agricultural Journal, 58: 688-690 
Singh, S. V., Mishra, A., Bisan, R. S., Malik, Y. P. and Mishra, A. 2000. Host preference of red pumpkin beetle, Aulacophora foveicollis and melon fruit fly, Dacus cucurbitae. Indian Journal of Entomology, 62: 242-246

Snow, J. W. 1988.Radiation Insect and Eradication in North America: An overview from screw worm to boll worm. IAEA-SM-301/29

Vagras, R. I. 1989. Alternative egg collection system for mass production of the Mediterranean fruit fly (Diptera: Tephritidae). Journal of Economic Entomology, 77: 1064-1169

Wadud, M. A., Hossain, M. A and Islam, M. S. 2005. Sensitivity of the melon fruit fly, Dacus cucurbitae. Indian Journal of Entomology, 62: 242-246

White, I. M. and Elson-harris, M. M. 1994. Fruit Flies of Economic Significance: Their Identification and Bionomics, Commonwealth Agriculture Bureau International, Oxon, UK. pp. 1-601 
Table 1. Pupal duration after imposing gamma radiation at different pupal ages of oriental fruit fly Bactrocera dorsalis

\begin{tabular}{lll}
\hline \multirow{2}{*}{ Dose (Gy) } & \multicolumn{2}{c}{ Pupal duration in days (Mean \pm SE) } \\
\cline { 2 - 3 } & \multicolumn{1}{c}{ 5-day-old pupae } & \multicolumn{1}{c}{ 6-day-old pupae } \\
\hline Control & $8.00 \pm 1.00$ & $7.67 \pm 0.58$ \\
30 & $8.67 \pm 0.58$ & $8.33 \pm 0.58$ \\
40 & $9.33 \pm 0.58$ & $8.67 \pm 0.58$ \\
50 & $10.00 \pm 1.00$ & $9.67 \pm 0.58$ \\
60 & $11.33 \pm 1.52$ & $11.00 \pm 1.00$ \\
LSD $(0.05)$ & 1.65 & 1.29 \\
CV $(\%)$ & 9.88 & 8.07 \\
\hline
\end{tabular}

Means having the same letter in each column do not differ significantly at $\mathrm{p}<0.05$ by LSD test.

Table 2. Normal emergence, deformed flies and nonemegence percentage of oriental fruit fly Bactrocera dorsalis at different radiation doses

\begin{tabular}{cccc}
\hline Dose & Normal emergence $(\%)$ & Deformed flies $(\%)$ & nonemegence flies $(\%)$ \\
\hline Control & 93.12 & 1.59 & 5.29 \\
30 & 87.67 & 4.59 & 7.73 \\
40 & 58.08 & 18.38 & 23.55 \\
50 & 16.34 & 15.35 & 68.31 \\
60 & 0.00 & 5.55 & 94.44 \\
\hline $\operatorname{LSD}(0.05)$ & 6.43 & 8.48 & 11.25 \\
$\mathrm{CV}(\%)$ & 6.60 & 49.54 & 14.99 \\
\hline
\end{tabular}

Means having the same letter in each column do not differ significantly at $\mathrm{p}<0.05$ by LSD test. 
Table 3. Number of pupae produced from different mating ratios of unirradiated and irradiated males with the virgin females (300) of oriental fruit fly Bactrocera dorsalis in field cage trials

\begin{tabular}{cccccccc}
\hline \multirow{2}{*}{ Ratios } & \multicolumn{7}{c}{ Adult age (days) } \\
\cline { 2 - 7 } & 14 th & 15 th & 16 th & 17 th & 18 th & 19 th & 20 th \\
\hline Control & $857.00 \pm 7.55$ & $905.67 \pm 5.69$ & $826.00 \pm 20.07$ & $871.33 \pm 27.06$ & $937.00 \pm 18.25$ & $875.33 \pm 14.57$ & $902.00 \pm 10.00$ \\
$1: 02$ & $660.33 \pm 10.02$ & $587.33 \pm 11.68$ & $578.67 \pm 16.29$ & $658.67 \pm 26.31$ & $598.67 \pm 22.01$ & $566.00 \pm 5.29$ & $653.33 \pm 21.73$ \\
$1: 05$ & $230.67 \pm 15.14$ & $243.33 \pm 4.62$ & $193.00 \pm 25.00$ & $217.33 \pm 24.66$ & $199.67 \pm 37.98$ & $236.33 \pm 21.22$ & $185.67 \pm 28.04$ \\
$1: 09$ & $44.00 \pm 7.21$ & $64.33 \pm 22.59$ & $52.67 \pm 4.51$ & $67.67 \pm 19.01$ & $65.00 \pm 9.54$ & $74.00 \pm 2.65$ & $47.67 \pm 11.59$ \\
\hline LSD (0.05) & 19.99 & 24.02 & 41.44 & 52.97 & 53.94 & 28.42 & 43.18 \\
CV (\%) & 2.23 & 2.67 & 5.03 & 5.84 & 6.00 & 3.25 & 4.83 \\
\hline
\end{tabular}

Means having the same letter in each column do not differ significantly at $\mathrm{p}<0.05$ by LSD test.

Table 4. Percent normal emergence of oriental fruit fly Bactrocera dorsalis from different mating ratios in field cage trials

\begin{tabular}{cccccccc}
\hline \multirow{2}{*}{ Ratios } & \multicolumn{7}{c}{ Adult age (days) } \\
\cline { 2 - 7 } & 14 th & 15 th & 16 th & 17 th & 18 th & 19 th & 20th \\
\hline control & 99.96 & 99.93 & 99.43 & 99.84 & 99.71 & 99.60 & 99.70 \\
$1: 02$ & 97.53 & 96.87 & 97.31 & 96.68 & 96.82 & 97.55 & 97.96 \\
$1: 05$ & 68.42 & 68.86 & 51.49 & 73.50 & 67.88 & 72.65 & 66.45 \\
$1: 09$ & 52.61 & 56.54 & 55.11 & 59.04 & 58.54 & 55.03 & 54.88 \\
\hline LSD (0.05) & 1.02 & 6.51 & 29.80 & 6.62 & 3.39 & 5.90 & 3.42 \\
CV (\%) & 5.06 & 4.04 & 19.67 & 4.03 & 2.10 & 3.64 & 2.15 \\
\hline
\end{tabular}

Means having the same letter in each column do not differ significantly at $\mathrm{p}<0.05$ by LSD test. 
Table 5. Percent deformed flies of oriental fruit fly Bactrocera dorsalis from different mating ratios in field cage trials

\begin{tabular}{cccccccc}
\hline & \multicolumn{7}{c}{ Adult age( days) } \\
\cline { 2 - 7 } Ratios & 14 th & 15 th & 16 th & 17 th & 18 th & 19 th & 20 th \\
\hline control & 0.00 & 0.07 & 0.11 & 0.15 & 0.07 & 0.17 & 0.03 \\
$1: 02$ & 0.73 & 1.04 & 0.88 & 1.03 & 0.92 & 0.87 & 1.08 \\
$1: 05$ & 20.09 & 18.04 & 18.32 & 16.08 & 21.47 & 15.85 & 18.97 \\
$1: 09$ & 28.75 & 27.17 & 29.25 & 28.64 & 29.86 & 26.95 & 25.84 \\
\hline LSD & & & & & & & \\
$(0.05)$ & 2.21 & 4.20 & 2.52 & 5.43 & 1.71 & 4.89 & 2.33 \\
CV (\%) & 8.94 & 18.17 & 10.41 & 23.68 & 6.55 & 22.34 & 10.18 \\
\hline
\end{tabular}

Means having the same letter in each column do not differ significantly at $\mathrm{p}<0.05$ by LSD test.

Table 6. Percent nonemergence of oriental fruit fly Bactrocera dorsalis from different mating ratios in field cage trials

\begin{tabular}{cccccccc}
\hline & \multicolumn{7}{c}{ Adult age( days) } \\
\cline { 2 - 7 } Ratios & 14 th & 15 th & 14 th & 17 th & 14 th & 19 th & 14 th \\
\hline control & 0.04 & 0.00 & 0.45 & 0.00 & 0.21 & 0.10 & 0.26 \\
$1: 02$ & 1.72 & 2.07 & 1.87 & 2.27 & 2.24 & 1.56 & 0.94 \\
$1: 05$ & 12.05 & 13.08 & 12.18 & 12.40 & 10.62 & 11.49 & 14.56 \\
$1: 09$ & 18.61 & 16.36 & 15.63 & 12.31 & 11.58 & 18.01 & 18.48 \\
\hline LSD $(0.05)$ & 2.50 & 4.07 & 3.66 & 5.40 & 3.52 & 1.89 & 3.08 \\
CV $(\%)$ & 15.43 & 25.85 & 24.34 & 10.08 & 28.56 & 12.17 & 17.99 \\
\hline
\end{tabular}

Means having the same letter in each column do not differ significantly at $\mathrm{p}<0.05$ by LSD test. 


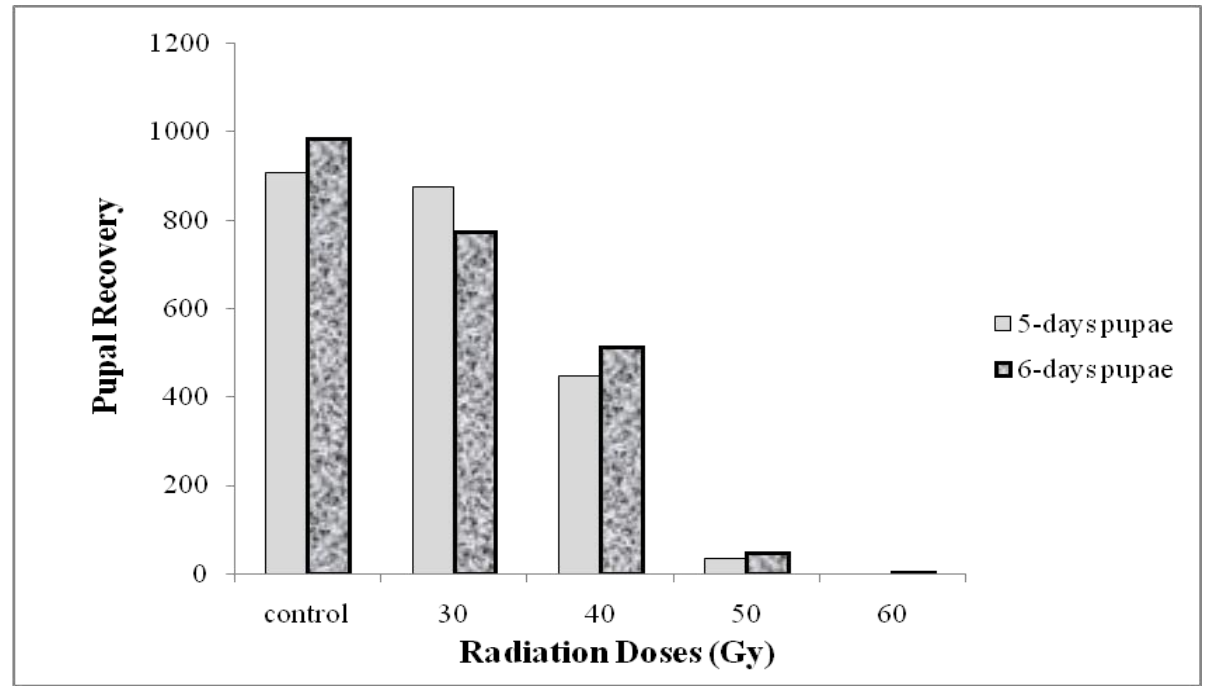

Figure 1. Mean number of pupal recovery of oriental fruit fly, Bactrocera dorsalis irradiated at different doses (Gy) of gamma radiation 